\title{
ANALYSIS OF ALTERNATIVES: AN EFFICIENT TOOL IN MANAGING FORCE MODERNIZATION PROJECTS
}

\author{
Venelin GEORGIEV
}

\begin{abstract}
Analysis of alternatives is a systematic method to deal with the problem of selecting a reliable variant and a common approach to the efficient allocation of limited resources among the competing candidate alternatives. In this method, the alternatives for meeting the existing needs are studied by assessment of the quantitative effects and costs for each of the considered alternatives. This becomes the objective that is approached by means of mathematical, economic, statistics, and other scientific methods. The analysis of alternatives is a process that includes definition of goals, main parameters, assumptions and constraints for the analysis; preparation of a list of considered alternatives; collection of data into a database and evaluation of the effects and cost for each alternative; performing sensitivity analysis; and reporting the final results from the analysis. The practical realization of the analysis of alternatives is related to the elaboration of a plan for the analysis, whose content is presented in this article.
\end{abstract}

Keywords: Analysis of Alternatives, Primary and Secondary Analysis, Measures of Effectiveness and Measures of Performance, Sensitivity Analysis, "Cost-Effectiveness” Analysis.

In conditions of market economy and budget limitations in the area of defense and security, the Armed Forces do not have sufficient financial resources to acquire the armaments necessary to meet the requirements of new missions, goals, and tasks. The existing limitations entail decision making for selection of a rational approach to acquire the required operational capabilities and to determine the actual projects for modernization to be funded with limited financial resources. There is a real necessity for a variant/ tool that enables the decision makers to choose from various considered alternatives the one with the highest efficiency, the realization of which involves rational and acceptable risk and expenditures. One such method is the Analysis of Alternatives (AoA) approach that could be defined as a systematic method for studying the problems of selection of alternatives and a common approach to the effective al- 
location of the limited resources among the competing needs. In this method, the alternatives for meeting existing needs are studied by assessing the quantitative effects and costs for each of the considered alternatives. This becomes the objective that is tackled by means of mathematical, economic, statistical, and other scientific methods which compare and rank the different alternatives. In the AoA approach the effects and costs for the alternatives are estimated for their entire life cycle.

The practical realization of the AoA approach requires theoretical knowledge summarized in this article. The theoretical background of AoA is supported in the article by an example of the application of the method within the area of research for selection of the most suitable multi-role fighter for the Air Forces. This example serves only as an illustration of a possible practical implementation of the AoA approach as a decision support tool and the results from this example are only illustrative. Furthermore, results presented in the article are not final due to the fact that the analysis has not been yet completed.

It is possible to use the AoA approach in other decision-making processes for performing portfolio selection of projects, for which there exist more than one possible and reliable alternative that satisfy defined needs and requirements.

In case there is only one alternative, which satisfies project requirements, comparison of the effects and life cycle costs is impossible and practically excludes the necessity of performing AoA.

From a practical point of view, for the realization of all projects for modernization of the Armed Forces, including the project for procurement of a new multi-role fighter for the Air Force, there are more than one possible and reliable alternatives and this fact entails the necessity to implement AoA for each of the projects.

AoA is one of the possible methods that support the Armed Forces management and the decision-making process, in particular. One of its main advantages is the possibility to represent in an understandable manner the mission requirements, the possible decisions, and the expected effects and financial terms of the different alternative decisions. Another advantage of the AoA is the possibility to compare different alternatives for realization of the projects on an equal basis. AoA makes it possible to assess the alternatives and goals in terms of costs, effects, and existing risk and when it is necessary they can be examined more precisely using sensitivity analysis.

The process of application of the AoA approach to the example of choosing a new multi-role fighter for the Bulgarian Air Forces consists of seven phases.

The first phase includes definition of goals and main parameters for the implementation of the AoA approach. Precise, clear and, if possible, quantitative definition of the AoA goals is being performed in order to achieve adequate realization of the project 
requirements. This is an important step in the implementation of the AoA approach due to the fact that without a clear vision for the issue what has to be examined, it will not be possible to reach the desired final results. Improperly defined goals can mislead the users of the AoA results and make them think that a particular decision has already been made; and that AoA is not performed for finding the most rational and economically justified decision, which satisfies the existing needs and requirements.

The AoA's goal for the example considered in this article could be defined as analysis of alternatives for acquiring required operational capabilities (ROC) for performing Air Force missions and tasks through procurement of multi-purpose aircraft or modernization of the current fleet, which possess the necessary capabilities for the implementation of the new missions and tasks, such as air superiority, air interdiction, close air support, kill boxing, reconnaissance, etc.

The analysis of alternatives approach includes the following common parameters.

Scope of Analysis

The main factors that influence the scope of the analysis are the defined needs and requirements that have to be met, the period of time for evaluation and the efforts and expenditures necessary for AoA realization. Whether the analysis will be primary or secondary depends on the nature of the needs. Primary analysis is performed when an alternative (proposal) for satisfying the existing needs with smaller amount of resources is examined. That means, when existing needs were satisfied in a particular way but at the same time there is a better alternative serving the same purpose. Secondary analysis is performed when new needs/ requirements have to be met or when the existing tools cannot satisfy the identified new needs any longer (refer to Figure 1$)^{1}$

For the purposes of the analysis of alternatives approach, the main characteristics of the project considered in this article makes it possible to classify the analysis as secondary - it is required to satisfy new needs of the Air Force.

\begin{tabular}{|c|c|c|c|}
\hline $\begin{array}{l}\text { Current fleet does not } \\
\text { meet the requirements } \\
\text { imposed by the Force } \\
\text { Goals Agreement }\end{array}$ & $\square$ & $\begin{array}{l}\text { Purchase of new multi- } \\
\text { purpose air craft } \\
\text { - Modernization of current } \\
\text { fleet }\end{array}$ & $\sqrt{\cdot \begin{array}{l}\text { Secondary } \\
\text { analysis }\end{array}}$ \\
\hline
\end{tabular}

Figure 1: Secondary Analysis of Alternatives. 


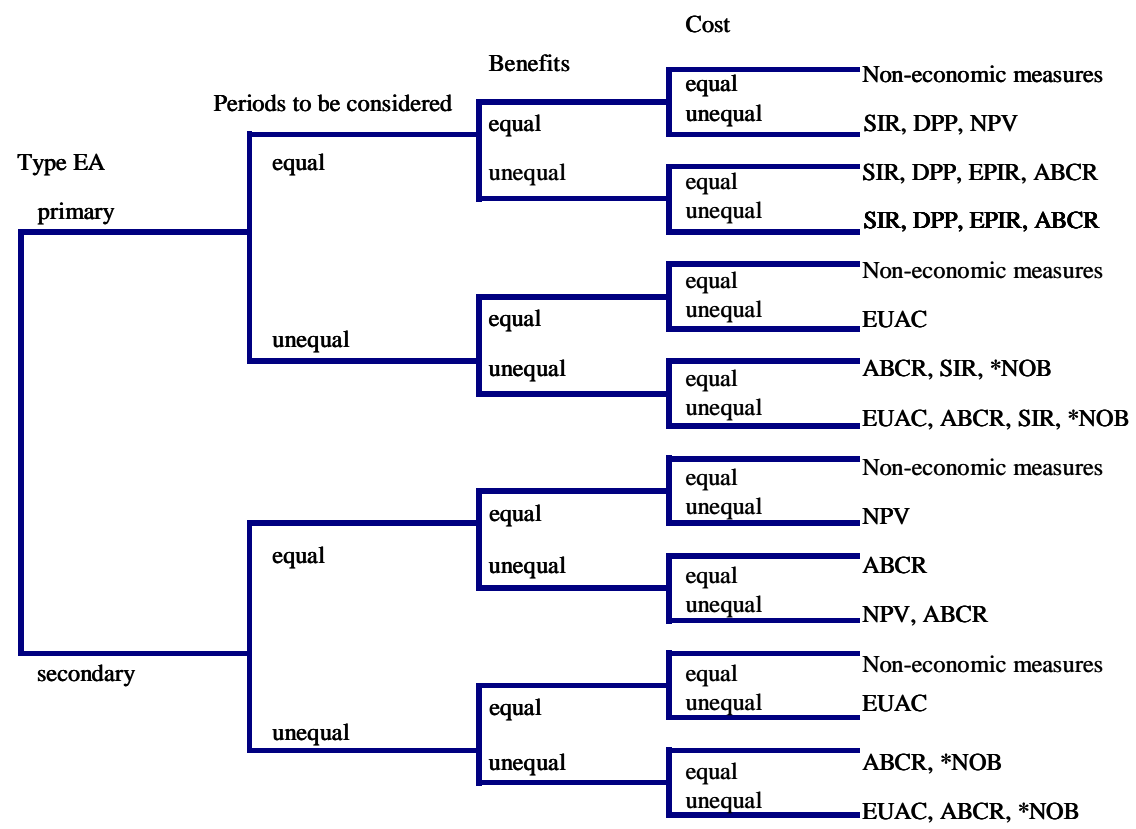

Figure 2: A Possible Chart for Choosing Methods for AoA Implementation.

\section{Method for Comparison of the Existing Alternatives}

There are different methods to compare competing alternatives; however, the most frequently applied ones are NPV, DPP, SIR, etc. The main factors that determine the method to be used for the particular project and its analysis are the amount of financial resources available, the effects/ benefits obtained by means of the examined alternatives and the duration of the period of analysis. There are various types of specific charts that can be used for the purpose of determining the method for comparison in the AoA approach. An example of such a type of chart is shown in Figure 2. ${ }^{2}$

Due to the fact that the size of the expected spending and the effects are different for the different alternatives considered in the example here, but the duration of the period for analysis is the same for each of the alternatives, the methods suitable for comparison of the examined alternatives are NPV or ABCR (refer to Figure 3).

Main Time Characteristics for the Analysis

The year of discounting the spending for the examined alternatives has been assumed as a starting point for the AoA's time period. This period includes several key points, such as mission's life, base year, lead-time, etc. Mission's life is a period over which the asset is needed. Base year is the year to which costs and benefits for the alterna- 


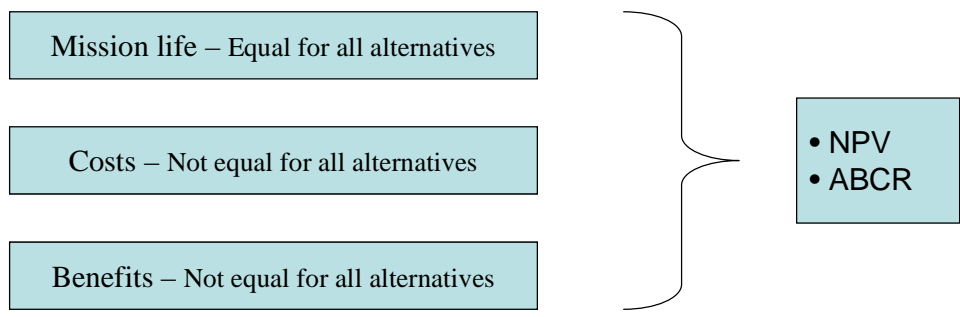

Figure 3: Choosing a Useful Method for Comparison of the Considered Alternatives.

tives will be discounted. Start year is the year in which initial investments for the considered alternatives are made. Lead-time is the time between the beginnings of start year to the beginning of the economic life of an asset for each of the examined alternatives.

\section{Measures of Effectiveness (MoE) and Measures of Performance (MoP)}

Measures of Effectiveness (MoE) represent the customers' view, usually assuming a qualitative nature. They describe the customers' expectations of a product, project or system. MoE can be represented in the form of a hierarchical diagram (see Figure 4). ${ }^{3}$ Each horizontal level in the hierarchy represents $100 \%$ of the effectiveness or performance. Weights can be attached to each design requirement. Evaluation of alternative designs can be made through the use of a method such as the weighted objective decision matrix or similar methods. The use of MoE enables the experts who perform the analysis to make the right decision and to propose the best alternative for solving the problem in consideration.

For the purposes of the analysis of alternatives for the example considered in this article, the following measures could be used as measures of effectiveness - survivability, vulnerability, cost, weapon system features, armament flexibility, exploitation, and upgrade.

The measures of performance express the producers' opinion for the project products. They represent the technical specifications of these products. ${ }^{4}$ As a rule, the measures

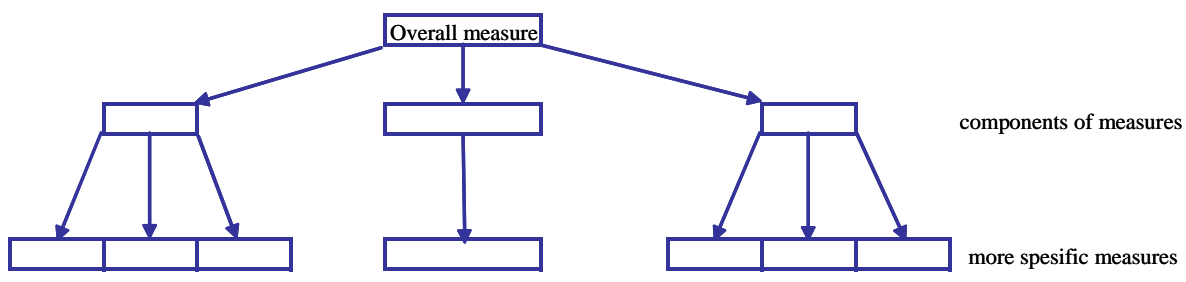

Figure 4: The MoE’s Hierarchy Diagram. 


\begin{tabular}{|c|c|c|}
\hline \multicolumn{3}{|c|}{ Specification } \\
\hline 1 & Maximum speed & $2000-2300 \mathrm{~km} / \mathrm{h}$ \\
\hline 2 & Maximum altitude & $15000-18000 \mathrm{~m}$. \\
\hline 3 & Range & $2000-2500 \mathrm{~km}$. \\
\hline 4 & G-max & 9Gs \\
\hline 5 & Combat radius & $500 \mathrm{~km}$. \\
\hline 6 & Combat endurance & $>2 \mathrm{~h}$. \\
\hline 7 & Weight - empty & $15-20$ tons \\
\hline 8 & Payload & $3-5$ tons \\
\hline 9 & Maximum wing loading & up to 4 tons \\
\hline 10 & Thrust - weight ratio & $0.95-1.2$ \\
\hline 11 & Reverse thrust of power plant & yes \\
\hline 12 & Active power thrust vector control & yes \\
\hline 13 & Max Vertical velocity & $300-350 \mathrm{~m} / \mathrm{s}$ \\
\hline 14 & Supersonic cruising speed & yes \\
\hline 15 & Takeoff and landing distance & $750-1000 \mathrm{~m}$ \\
\hline 16 & Navigation/weapon delivery system & yes \\
\hline 17 & Weapons loads & up to 8 pylons \\
\hline 18 & Systems for self-defense & yes \\
\hline 19 & Inoperability with land and air based systems & yes \\
\hline 20 & Ability to transfer data in real time at a distance more than $150 \mathrm{~km}$ & yes \\
\hline 21 & Container for air reconnaissance & yes \\
\hline 22 & Container for electronic warfare and reconnaissance & yes \\
\hline 23 & Aerial refueling & yes \\
\hline 24 & Catapult system at Vo and Ho & yes \\
\hline 25 & Exploitation system & yes \\
\hline
\end{tabular}

Figure 5: Specification for the Desired Fighter.

of performance are quantitative characteristics and their purpose is to demonstrate what the producers have done to reach the requirements of the customers by producing the desired products (see Figure 5).

For MoP in the analysis presented here, applying the method of expert assessment leads to the estimation of the significance of the characteristics needed to perform the required missions and tasks shown in Figure 5. The results from this assessment are as presented in Table $1 .^{5}$

At probability $P=0.1$ and degree of freedom $n-1=25-1=24$, the tabulated value of the Pearson's criteria is 61.1. Comparing the analytical and tabulated values of the Pearson's criteria shows: $V=63>61.1=\chi^{2}$. This comparison gives high confidence to conclude that with a probability of no less than $90 \%$, we can assume that there is a strong correlation between the opinions of the experts and thus we could use the results for the AoA. Based on the results from the expert assessment, charts for the significance of the MoP are drawn using different methods (Figures 6 and 7). 
Table 1: Statistical Results.

\begin{tabular}{|c|c|}
\hline $\begin{array}{l}\text { Ranking Total: } \\
\sum_{i=1}^{n} X_{i}=\frac{1}{2} n(n+1)=\frac{1}{2} 25.26=325\end{array}$ & $\begin{array}{l}\text { Corrector: } \\
U_{j}=\frac{1}{12} \sum_{i=1}^{n} u_{i j}\left(u_{i j}^{2}-1\right)=25.5\end{array}$ \\
\hline $\begin{array}{l}\text { Parameter } a \text { : } \\
a=\frac{1}{2} m(n+1)=130\end{array}$ & $\begin{array}{l}\text { Parameter } S_{d} \text { : } \\
S_{d}=\sum_{i=1}^{n}\left(\sum_{j=1}^{m} X_{i j}-a\right)^{2}=34059\end{array}$ \\
\hline $\begin{array}{l}\text { Coefficient of concordance: } \\
W=\frac{S_{d}}{\frac{1}{12} m^{2} n(n-1)-m \sum U_{j}}=0.2625\end{array}$ & $\begin{array}{l}\begin{array}{l}\text { Analytical value of Pearson's crite- } \\
\text { ria: }\end{array} \\
V=\frac{S_{d}}{\frac{1}{12} m n(n+1)-\frac{1}{n-1} \sum U_{j}}\end{array}$ \\
\hline
\end{tabular}

The second phase of the AoA's approach includes making a complete list of alternative decisions to solve the problem under consideration. This list includes all rational and reliable alternatives and even those alternatives that for some reason cannot be realized at this moment. It is noteworthy that an incomplete list of alternatives can be a reason to question the validity of the AoA approach. The alternatives that are not

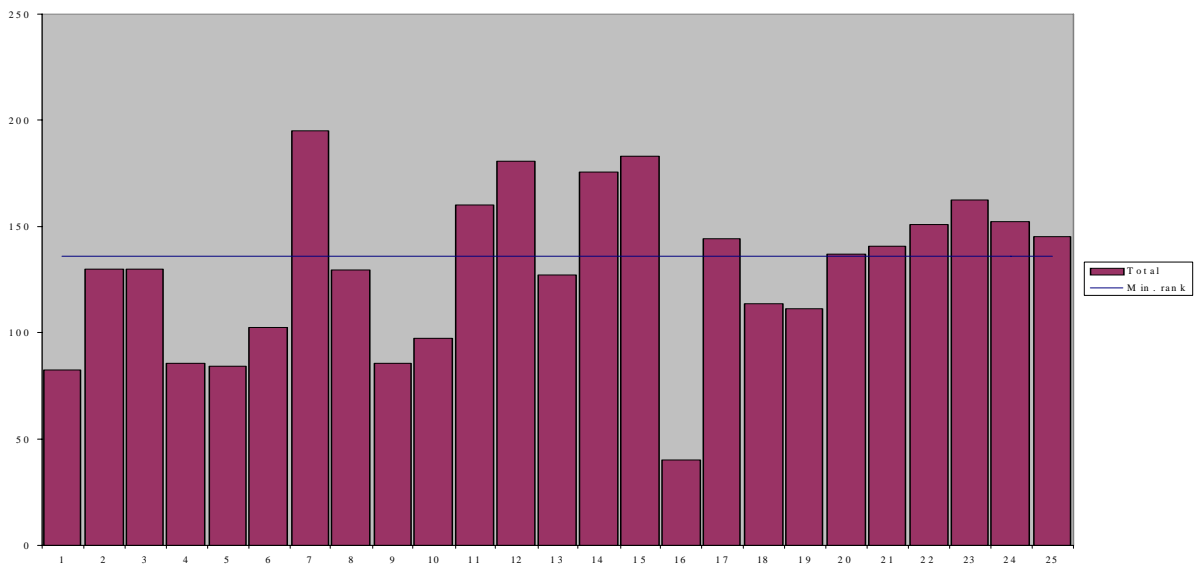

Figure 6: Histogram of the MoP. 


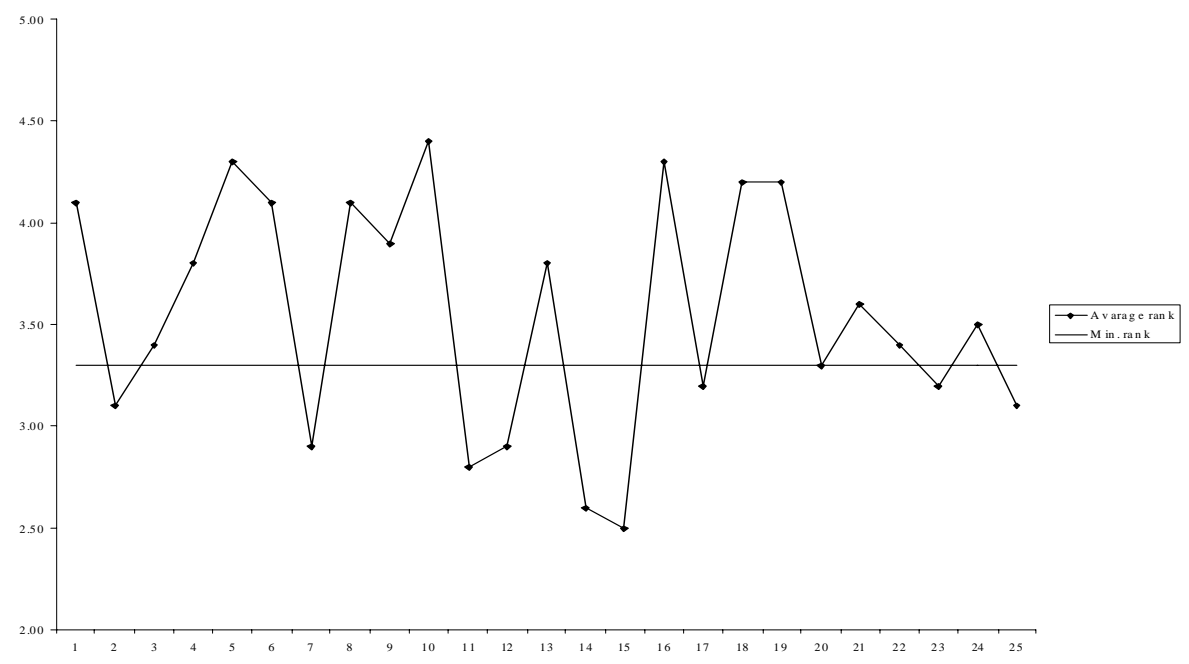

Figure 7: Applying the Method of Semantic Differential.

rational and reliable enough at the moment of analysis should be discussed and documented within the AoA approach, but it is not necessary to include them in the future work on cost and effects estimation. As a rule, one alternative is rational and reliable when it completely satisfies the stated requirements and its realization is possible at the period of analysis.

Possible alternatives for realization of the projects for modernization could be defined as producing, purchasing or leasing new types of armaments, purchasing or leasing "second-hand" armaments and modernization of the existing types of armaments. In practice, the realization of the projects for modernization of the Armed Forces is possible by combination of different approaches, which was mentioned above. For the project considered as an example in this article, the list of possible alternatives includes modernization of the existing types of fighters, MiG-21 or MiG29, as well as purchase of new or "second hand" fighters, for example F-16, F-18, and Gripen. The first two alternatives from the list (modernization of the existing types of fighters) are not rational and reliable enough and they should be only documented as part of the analysis. The other alternatives have to proceed to the next steps of the AoA, as it is shown in Figure 8.

The third phase of the AoA's approach includes the definition of assumptions and constraints. In general, the analysis of alternatives is performed in conditions of lack of complete information, which imposes assumptions and constraints to be defined. The goal is to reduce the extent of uncertainty in the analysis. Sometimes, the assumptions and constraints for the AoA are defined before choosing the examined al- 


\begin{tabular}{|c|c|c|c|c|c|}
\hline \multicolumn{2}{|r|}{ Specification } & \multirow{2}{*}{$\begin{array}{l}\text { Requirements } \\
\text { yes }\end{array}$} & \multirow{2}{*}{\begin{tabular}{|l}
$\begin{array}{c}\text { Alt_1 } \\
\text { F-18 E/F }\end{array}$ \\
yes
\end{tabular}} & \multirow{2}{*}{$\begin{array}{r}\text { Alt_2 } \\
\text { F-16 C/D }\end{array}$} & \multirow{2}{*}{\begin{tabular}{|c} 
Alt_3 \\
GRIPEN \\
yes
\end{tabular}} \\
\hline 1 & Navigation/weapon delivery system & & & & \\
\hline & Maximum speed & $2000-2300 \mathrm{~km} / \mathrm{h}$ & 1900 & 2142 & 2126 \\
\hline 3 & Combat radius & $500 \mathrm{~km}$. & 722 & 1252 & 800 \\
\hline 4 & G-max & 9Gs & 9 & 9 & 9 \\
\hline 5 & Maximum wing loading & Min. 4 tons & 7,7 & 7,2 & 6,5 \\
\hline 6 & Thrust - weight ratio & $0.95-1.2$ & - & 1,1 & - \\
\hline 7 & Combat endurance & $>2 \mathrm{~h}$ & 2,5 & - & - \\
\hline 8 & Inoperability with land and air based systems & yes & - & - & - \\
\hline 9 & Systems for self-defense & yes & yes & yes & yes \\
\hline 10 & Max Vertical velocity & $300-350 \mathrm{~m} / \mathrm{s}$ & - & 294 & 80 \\
\hline 11 & Payload & $3-5$ tons & - & - & - \\
\hline 12 & Maximum altitude & $15000-18000 \mathrm{~m}$. & 15,2 & 15,3 & 19 \\
\hline 13 & Range & $2000-2500 \mathrm{~km}$. & 3700 & 4215 & 3300 \\
\hline 14 & Ability to transfer data in real time at a distance more than $150 \mathrm{~km}$ & yes & - & - & - \\
\hline 15 & Container for air reconnaissance & yes & yes & - & - \\
\hline 16 & Weapons loads & Min. 8 pylons & 9 & 9 & 8 \\
\hline 17 & Exploitation system & yes & yes & yes & yes \\
\hline 18 & Container for electronic warfare and reconnaissance & yes & yes & - & - \\
\hline 19 & Catapult system at Vo and Ho & yes & yes & yes & yes \\
\hline 20 & Reverse thrust of power plant & yes & yes & - & yes \\
\hline 21 & Aerial refueling & yes & yes & yes & yes \\
\hline 22 & Supersonic cruising speed & yes & yes & - & yes \\
\hline 23 & Active power thrust vector control & yes & yes & - & yes \\
\hline 24 & Takeoff and landing distance & $750-1000 \mathrm{~m}$ & $430 / 620$ & $450 / 650$ & $400 / 600$ \\
\hline 25 & Weight - empty & $15-20$ tons & 16,65 & 11,84 & 9,7 \\
\hline
\end{tabular}

Figure 8: List of Alternatives for the AoA used in the Article as an Example.

ternatives. The assumptions and constraints should determine the environment for the analysis in a way that ensures correct understanding for their influence on the final results from the AoA. This is extremely important for the people who will make the final decision.

For the example in this article, assumptions and constraints could be defined in the following areas - span of their life-cycle cost and effects, technical and tactical characteristics, cost for utilization and maintenance, etc.

The fourth stage of the AoA approach includes gathering data for the effects and cost of the examined alternatives, storing them into a database and executing the computations. The sources used for gathering data and the results from the computations should be correctly documented as they determine how precise the obtained results are. This phase of the AoA approach is the most critical part of the analysis. It needs sufficient time and has a major impact on the accuracy of the final results.

There are three main types of cost estimates:

- $\quad$ Detailed estimates - within 5 \% of actual costs;

- Good estimates - accuracy is about 10 \% of actual costs;

- Order of magnitude estimates - estimates differ from actual data by as much as $50 \%$.

The people performing the analysis should take into account all cost factors and expected effects from each of the examined alternatives in their association. They can 
use different methods for life cycle cost analysis:

- Engineering cost method used when there is detailed and accurate capital and operational cost data. It involves direct cost estimation of a particular cost element;

- Analogous cost method used when there is a high level of detail drawn on historical data from other asset of analogous size, technology, and operational characteristics;

- Parametric cost method used when an actual or historical cost data is limited around known parameters.

Each alternative is examined separately. Computations on the effects and cost for the alternatives may be performed using existing models, but it is also possible to make the computations using models developed by the people performing the analysis.

During the fifth stage of the AoA approach, comparison and ranking of the examined alternatives by means of cost-benefits analysis is completed. In the literature, this stage of the AoA is known as "the heart of the analysis." But, at the same time, the implementation of this stage could be the easiest part of the analysis if the previous four stages have been carried out precisely and completely. At this stage, three main criteria could be used for comparison and ranking of the examined alternatives:

- "Min" cost if the effects from the alternatives are equal;

- "Max" effects if the costs for the alternatives are equal;

- "Max" ratio effects/ cost if both are not equal for the alternatives.

These three criteria determine three types of links between costs and benefits of the examined alternatives - unequal cost and equal effects; equal cost and unequal effects; unequal cost and unequal effects.

When all considered alternatives have equal costs and equal effects, they are ranked using non-economic criteria. The possible cases of comparison of alternatives are shown in Figure 9. ${ }^{6}$

\begin{tabular}{|l|l|l|}
\hline \multicolumn{3}{|c|}{ Comparison of Alternatives } \\
\hline Costs & Benefits & Basis for Recommendation \\
\hline Equal & Unequal & Most benefits \\
\hline Unequal & Equal & Least cost \\
\hline Unequal & Unequal & Highest benefits to cost ratio \\
\hline Equal & Equal & Other factors \\
\hline
\end{tabular}

Figure 9: Possible Cases for Comparison of Alternatives. 
The sixth stage of the AoA approach includes performing sensitivity analysis. This specific type of analysis is made when the level of risk for the AoA is too high or when there is no considered alternative, which is obviously better than the others. The sensitivity analysis answers "what-if" questions and helps in finding results and final conclusions, which vary in broad intervals if some of the considered factors for the AoA such as cost, assumptions, constraints, or MoP have changed their parameters. The sensitivity analysis is performed mandatory for projects, whose results from the AoA do not show the best alternative for meeting the customer requirements. If a change in the value of some of the considered factors is a reason for change in the ranking of the considered alternatives, it means that the AoA is sensitive to this factor. The factors that demonstrate significant influence on the extent of the cost and effects for the alternatives form suitable basis for performing sensitivity analysis. An appropriate way to find these factors is to evaluate the influence of their change in percentage on the cost and effects for the alternatives. From practical point of view, all factors that are related to spending of size bigger than $20 \%$ from the total spending for the alternative have to be considered in the sensitivity analysis.

The sensitivity analysis is usually performed not for all alternatives but only for those ranked first and second and the investigated changes of the factors are in the same direction - an increase or a decrease. Performing sensitivity analysis and including the results from this analysis in the report for the AoA application provide the necessary information to the decision makers about the influence of existing uncertainties and risks on the final choice.

The seventh stage of the AoA consists of reporting of the results from the analysis and making recommendations to the decision makers. This is essential phase of the AoA approach - the best alternative has to be shown and recommendations for its implementation have to be formulated. The recommendations to the decision makers are extremely important because, sometimes, the ranking of the alternatives does not show clearly which alternative satisfies the requirements in the best possible way.

For almost all AoA for the Armed Forces modernization projects, the best alternative could be that with the lowest level of spending estimated for the period of analysis. This conclusion is correct due to the assumption that all examined alternatives are rational and reliable and they satisfy the stated requirements.

The final results from the application of the analysis of alternatives approach should be presented to the decision makers in the most convenient way for use and understanding. The report includes the goals of the AoA, the assumptions and constraints, the list of examined alternatives, the summarized database for life cycle costs and effects, the appropriate charts, and the results from sensitivity analysis. The report 
should end with conclusions and recommendations. And as an example, the content of the AoA report may consist of:

- Summary of the AoA application, including goals, alternatives, their ranking, conclusions, and recommendations. Here is the place of the assumptions and constraints made in the beginning of the analysis.

- Detailed analysis of the life cycle cost and effects for the considered alternatives. Here, within a table, are shown detailed effects and cost for each of the examined alternatives for each year of the period of analysis.

- Charts that illustrate the results from the analysis.

- The results from the sensitivity analysis including the examined factors and the arguments for their choice. Here, the results obtained after changing the parameters of the factors are presented.

The practical realization of the AoA approach is related to the development of appropriate plan and its implementation. The plan should contain a description of the approach for AoA implementation, the main tasks, and who is responsible for solving these tasks. Only as a suggestion, the content of the AoA implementation plan may include the following elements:

- Introduction that explains the origin of the problem in consideration, the goal and the scope of the analysis. The introduction includes description of the necessity to perform AoA. The level of detail for the AoA approach that is appropriate for the particular situation is defined.

- AoA main rules, which include the scenarios and threats leading to the definition of the requirements, assumptions and constraints for the analysis. The considered scenarios are extracted from the defense planning scenarios and they are enriched with specific details. The environmental factors, which influence the examined scenarios and tasks, such as climate and terrain, are important for the analysis and they are part of the AoA main rules. The AoA constraints are described as factors that limit the possible options for the AoA team. On the other hand, when they are correctly defined and if they truly represent the system characteristics, the constraints increase the probability of reaching the final goals of the analysis. Understandably, even the most precise analysis is realistic only within the constraints and if these constraints change, the AoA will lose accuracy and effectiveness. The assumptions are factors presumed to be true statements during the AoA planning process. The assumptions account for the uncertainty in analysis and this explains why they influence the level of risk in the AoA.

- The examined alternatives, which are presented describing their content, probability, expected benefits, operational and support concepts. The consid- 
ered alternatives are documented in the AoA implementation plan and their number could be changed during the AoA implementation. It is a normal practice to limit the number of rational and reliable alternatives in the beginning of the AoA. In the course of the AoA application, after receiving some new information, the AoA team could add new alternatives to be considered in the analysis. The number of considered alternatives could be controlled by avoiding similar alternatives and eliminating some of the alternatives using, for example, reasons such as unacceptable level of risk and life cycle cost.

- AoA implementation technology. The estimation of the expected benefits and life cycle costs for the examined alternatives presupposes knowledge of specific details from the area of operational and supporting concepts. The operational concepts describe in detail the conditions for use of each alternative in peacetime, during crisis and wartime. The supporting concepts describe the planned activities related to the realization of the considered alternatives in such areas as personal training, utilization and maintenance of the facilities and infrastructure, etc. The matrix for assessment of the examined alternatives is described in the AoA implementation plan, which in the majority of the cases is constructed and presented as a hierarchical system of mission tasks, $\mathrm{MoE}$, and MoP. The mission tasks describe the necessity of required new capabilities. The MoE could be viewed as a more detailed matrix that enables the quantitative assessment of tasks and missions' implementation. One or more MoE have to be defined for each mission task, which describe the effectiveness of each of the considered alternatives in performing a task. On the other hand, each MoE should support one or more mission tasks. As a rule, the MoP are quantitative characteristics and they are used when it is necessary to estimate the MoE. The AoA implementation plan presents the selected analytical approach for analysis of the effectiveness of the considered alternatives as a hierarchical system of mission tasks, MoE, MoP, scenarios, and treats. The chosen analytical approach defines the level of detail for the effectiveness analysis of the examined alternatives. This level depends on the number of considered scenarios, threats, and alternatives. The approach for life-cycle cost assessment of the considered alternatives is also detailed in the AoA implementation plan. The cost analysis is performed in parallel with the effectiveness analysis. In the cost analysis, the life-cycle cost for each of the examined alternatives is evaluated separately. When the costs for the alternatives are estimated in a long time period, the cost analysis is performed using discounting methods. It is important to emphasize that the life-cycle cost analysis is one of the most important parts of the AoA approach and it requires the knowledge and expertise of very well prepared experts. The results from the cost analysis are used together with the results from the effectiveness 
analysis for comparison of the examined alternatives, laying the foundation for comparison along different criteria. The final analytical part of the AoA implementation plan is related to planning of the comparison of the considered alternatives using "cost-benefit” criteria. In the majority of the analysis, the examined alternatives have different effects and life-cycle costs, a fact that poses the question how to determine whether marginal increase of effectiveness justifies the associated marginal cost. From practical point of view, it is impossible to find the perfect conditions when the effects and cost for the examined alternatives are absolutely equal due to the complex nature of the alternatives.

- Finally, the AoA implementation plan includes a description of the procedure for management of the AoA implementation. In general, a dedicated team with a designated leader performs the AoA implementation. The team consists of cautiously selected experts from different areas of the analysis. The AoA implementation team includes different working groups (WG), for instance WG for scenarios and threats; WG for definition of alternatives; WG for definition of operational and support concepts; WG for effectiveness analysis; WG for life-cycle cost analysis, etc. For the largest part of the AoA, the central role is played by the WG for effectiveness analysis and this group combines and relates the work of the other groups.

The plan for AoA implementation proposed in this article is not mandatory for each AoA; it provides and example to acquisition management experts. Obviously, each AoA has unique features which explains why the implementation plan for each particular AoA is unique, too.

As a conclusion, we could emphasize that the AoA is one of the most valuable tools that managers could and should use in conditions of market economy to manage the projects of their organizations. Using the AoA in practice requires knowledge of the respective theory, which will ensure that the results desired by the managers will be reached.

\section{Acknowledgement}

This paper reflects research on project SfP 981149 "Operations Research Support to Force and Operations Planning in the New Security Environment," sponsored by NATO's Scientific Affairs Division in the framework of the Science for Peace Program. 


\section{Notes:}

${ }^{1}$ Government Asset Management Committee, Life Cycle Costing Guideline (Sydney, New South Wales: Government Asset Management Committee, 2001).

${ }^{2}$ Life Cycle Costing.

${ }^{3}$ Measures of Effectiveness (MOE) and Measures of Performance, Design Methods Fact Sheet (University of Queensland, 2001).

${ }^{4}$ Measures of Effectiveness and Measures of Performance.

${ }^{5}$ E. Bojanov and I. Vuchkov, Statistical Methods for Modeling and Optimization of MultiFactor Objects (1999).

${ }^{6}$ Life Cycle Costing.

Colonel VENELIN GEORGIEV is a state expert in the Defense Acquisition Policy Section of the Armament and Equipment Policy Directorate at Bulgaria's Ministry of Defense. He holds a doctoral degree in the area of management and master degrees in the areas of technical, military and economic sciences. His experience is in the areas of program and project management of the defense resources, defense acquisition, and risk management. He is author of 30 articles in the field of defense and force management. 